\title{
American College of Cardiology/American Heart Association Lesion Complexity Score C
}

National Cancer Institute

\section{Source}

National Cancer Institute. American College of Cardiology/American Heart Association

Lesion Complexity Score C. NCI Thesaurus. Code C100039.

Diffuse (length greater than $2 \mathrm{~cm}$ ) or excessive tortuosity of proximal segment or extremely angulated segments greater than 90 degrees or total occlusions greater than 3 months old and/or bridging collaterals or inability to protect major side branches or degenerated vein grafts with friable lesions. (ACC) 